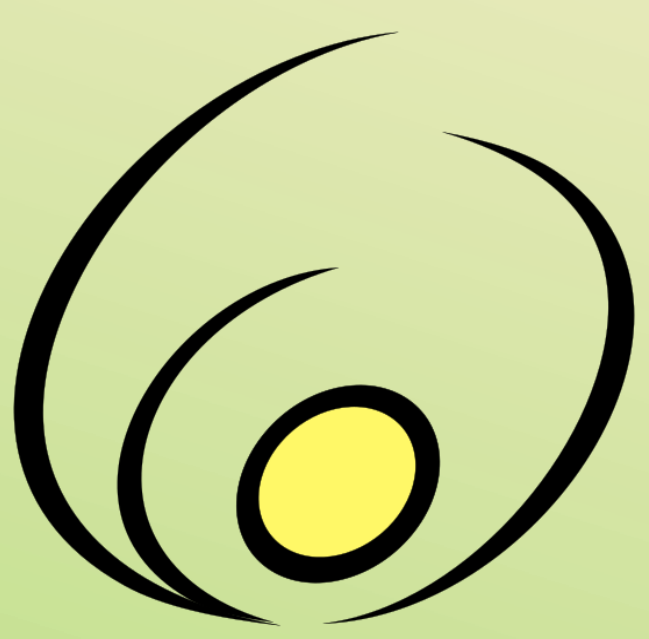

Fórum de

\section{Pró-Reitores}

\section{de Extensão}

\section{das Instituições}

\author{
Públicas de
}

\section{Educação Superior}

Brasileiras
Open access $\delta$ free available online

Revista Brasileira de Extensão Universitária

v. 8, n. 3, p. 143-148 set.- dez. 2017 e-ISSN 2358-0399

DOI: https://doi.org/10.24317/2358-0399.2017v8i3.5861

\title{
Educação em Saúde Bucal a Gestantes, Puérperas e Primeira Infância: Relato de Atividade de Extensão
}

\author{
Marcos Cezar Pomini ${ }^{1}$, Alana Taís Gawlik ${ }^{2}$, \\ Natali Pereira ${ }^{2}$, Amanda Rodrigues dos Santos ${ }^{2}$, \\ Bianca Rodrigues dos Santos², Jessyca Twany Demogalski³, \\ Nayara Silva de Gouvêa ${ }^{3}$, Fabiana Bucholdz Teixeira Alves ${ }^{4}$
}

Resumo: A abordagem educativa durante o período gravídico-puerperal e primeira infância é fundamental para que a mãe compreenda a influência das suas ações na saúde do bebê. O projeto de extensão "Educação em saúde bucal a gestante e na primeira infância" objetiva desenvolver atividades nos âmbitos educativos e preventivos com gestantes, puérperas e primeira infância. 0 projeto é desenvolvido em um hospital universitário do Sul do Brasil, e conta com a participação de docentes, mestrandos, residentes em odontologia hospitalar neonatal e acadêmicos de graduação em odontologia. O projeto atua em três frentes: a primeira, com as gestantes, onde é realizada prioritariamente a abordagem educativa no pré-natal odontológico, principalmente quanto à alimentação e higiene oral adequadas; a segunda frente, no puerpério imediato, com orientações de amamentação e sucção não-nutritiva; e a terceira frente, atuando na avaliação bucal, teste da linguinha e acompanhamento do bebê até um ano de vida. Os autores constataram que o projeto auxilia na criação de um vínculo entre as gestantes/puérperas e os acadêmicos/residentes, que resulta em uma melhora na aquisição e manutenção de saúde bucal da mãe e do bebê. Assim como auxilia o desenvolvimento e capacitação dos participantes para o atendimento a este público.

Palavras-chave: Assistência à Saúde, Educação em Odontologia, Gestantes, Recémnascido, Odontopediatria, Extensão Universitária

Content shared under Creative Commons Attribution 3.0 Licence CC-BY

\footnotetext{
1 Graduando em Odontologia, Universidade Estadual de Ponta Grossa - UEPG, Departamento de Odontologia, Campus Uvaranas, marcospomini@outlook.com Av Gal. Carlos Cavalcanti, 5775, Ap. 20, Bloco D, Uvaranas, 84030-000 - Ponta Grossa - PR, Brasil (autor para correspondência)

2 Graduandos em Odontologia, UEPG, Campus Uvaranas, Ponta Grossa - PR, a.t.g@hotmail.com, nataligorges@gmail.com, amanda.dos.santos@hotmail.com, brodriguesdossantos@hotmail.com

3_Cirurgiã-dentista. Residente em Odontologia Hospitalar Neonatal no Hospital Universitário Regional dos Campos Gerais - HURCG, email: jessycademogalski91@gmail.com, nayaraodontologia13@gmail.com

4_Professora Adjunto do Curso de Odontologia da Universidade Estadual de Ponta Grossa, Departamento de Odontologia, Campus em Uvaranas, Ponta Grossa, PR, email: fabi.teixeira@uol.com.br
} 


\title{
Oral Health Education in Pregnant and Puerperal Women and Infants: Report of Extension
}

\section{Activity}

\begin{abstract}
The educational approach during the pregnancy-puerperal period and early childhood are fundamental for the mother to understand the influence of her actions on the health of the baby. The extension project "Oral health education in pregnant and early childhood" aims at developing activities in the educational and preventive areas with pregnant and puerperal women, and early childhood. The project is developed in a university hospital in southern Brazil and counts with the participation of professors, masters, residents in neonatal hospital dentistry and dental undergraduate students. The project operates on three approaches: the first, with the pregnant women, where the educational approach in dental prenatal care is carried out as a priority, especially regarding feeding and oral hygiene; the second approach, in the immediate puerperium, with guidelines for breastfeeding and non-nutritive sucking; and the third, with oral evaluation, Neonatal Tongue Screening Test and baby follow-up up to one year. The authors found that the project helps to create a bond between the pregnant/ puerperal women and the student/resident, which results in an improvement in the acquisition and maintenance of oral health of mothers and babies. It also helps the development and training of participants to attend this public.
\end{abstract}

Keywords: Health Care, Dental Education, Pregnant Women, Infant Newborn, Pediatric Dentistry, University Extension

\section{Educación en salud bucal a gestantes, puérperas y primera infancia: relato de actividad de} extensión

Resumen: El enfoque educativo durante el período gravídico-puerperal y la primera infancia son fundamentales para que la madre comprenda la influencia de sus acciones en la salud del bebé. El proyecto de extensión "Educación en salud bucal a la gestante y en la primera infancia" tiene por objeto desarrollar actividades en los ámbitos educativos, preventivos y curativos con gestantes, puérperas y primera infancia. El proyecto se desarrolla en un hospital universitario del sur de Brasil, y cuenta con la participación de docentes, residentes en odontología hospitalaria neonatal y estudiantes pregrados e postgrados de odontología. El proyecto actúa en tres frentes: la primera, con las gestantes, donde se realiza prioritariamente el abordaje educativo en el prenatal odontológico, principalmente en cuanto a la alimentación e higiene oral adecuadas; la segunda frente, en el puerperio inmediato, con orientaciones de lactancia y succión no nutritiva; y la tercera, con la evaluación bucal y del frenillo de la lengua, hasta un año de vida. Los autores constataron que el proyecto auxilia en la creación de un vínculo entre las gestantes/puérperas y los estudiantes/residentes, que resulta en una mejora en la adquisición y mantenimiento de salud bucal de la madre y del bebé. Así como auxilia el desarrollo y capacitación de los participantes para la atención a este público.

Palabras-clave: Atención de Salud, Educación en Odontología, Mujeres Embarazadas, Recién Nacido, Odontología Pediátrica, Extensión Universitaria

\section{Introdução}

A Promoção da Saúde é definida pela carta de Ottawa como a capacitação do estado, indivíduos e comunidades de modificarem os determinantes de saúde em benefício da qualidade de vida populacional (BUSS, 2000). Inserida em um conceito amplo de saúde, as ações de saúde bucal devem ser integradas com as demais práticas de saúde coletiva, atuando assim na redução de fatores de risco, os quais constituem uma ameaça para o bem-estar físico e psicológico dos indivíduos (REIS et al., 2010). O período gravídico-puerperal é um momento de alterações físicas, fisiológicas, psíquicas e sociais que tornam as mulheres mais receptivas às mudanças $\mathrm{e}$ ao processamento de informações. Essas mudanças podem ser revertidas em benefício do bebê e seu núcleo familiar (MOIMAZ et al., 2007, REIS et al., 2010).

No entanto, as gestantes frequentemente apresentam certa resistência frente ao atendimento ambulatorial odontológico, grande parte devido à popularização de mitos e crenças culturais de que esse possa prejudicar o desenvolvimento do bebê (MOIMAZ et al., 2007). A literatura tem apontado que os principais fatores que contribuem para a disseminação de mitos são o desconhecimento da gestante e de outros profissionais sobre a importância da saúde bucal para o desenvolvimento do feto (BASTIANI et al., 2010).

Outro fator que pode estar contribuindo é a capacitação insuficiente do cirurgião-dentista no manejo clínico de 
gestantes. A literatura tem demonstrado que existe uma lacuna na formação de cirurgiões-dentistas que proporciona desconhecimento ou medo quanto à abordagem a esse público, levando a postergação de procedimentos ou não intervenção de maneira resolutiva (SANTOS NETO et al, 2012). Atualmente, os cursos de odontologia têm sofrido mudanças em suas grades curriculares que visam acentuar os cuidados na atenção primária e a formação mais humanística, generalista, crítica e reflexiva dos cirurgiões-dentistas (SILVEIRA; GARCIA, 2015). Essa mudança de foco na formação dos cirurgiões-dentistas, voltada mais para a prevenção de saúde, pode contribuir para o atendimento odontológico à gestante.

Portanto, o presente projeto de extensão, cuja ação temática é "Educação em saúde bucal à gestante e na primeira infância", busca o aprimoramento profissional de acadêmicos de odontologia visando o tripé ensinopesquisa-extensão, assim como a promoção de saúde bucal e geral de gestantes, puérperas e primeira infância. Deste modo, o objetivo deste artigo foi relatar as ações em níveis educacionais e preventivos, desenvolvidos em três momentos distintos: pré-natal odontológico, puerpério imediato, e avaliação do recém-nascido (RN), de maneira a incentivar iniciativas semelhantes em outras instituições de ensino ou ambulatórios de saúde.

\section{Métodos}

\section{Fundação do Grupo e Funcionamento}

Tendo em vista que quanto mais cedo for realizada a abordagem odontológica educativa e preventiva, os índices de problemas bucais, como a doença cárie e a perda dental precoce, poderiam ser reduzidos na população como um todo. A partir desse princípio, o projeto "Educação em saúde bucal à gestante e na primeira infância" teve início em agosto de 2016, sendo executado com a totalidade das gestantes de alto risco que realizam o pré-natal em um hospital universitário regional. Atualmente, o projeto conta com a participação da docente responsável, uma mestranda em Ciências da Saúde, duas residentes em Odontologia Hospitalar Neonatal, sete alunos de especialização em odontopediatria e treze acadêmicos de graduação em odontologia da Universidade Estadual de Ponta Grossa UEPG.

O início deu-se com a criação de um grupo de estudo, o qual visou obter o referencial teórico para o desenvolvimento do projeto, e elaborar protocolos e materiais educativos para a atuação dos participantes. Inicialmente, foram desenvolvidos três materiais educativos, a saber: uma cartilha para a gestante, com informações sobre o pré-natal odontológico e a relação entre saúde bucal e saúde da gestante e do bebê; um banner autoexplicativo, fixado na sala de espera do hospital; e um vídeo autoexplicativo, reproduzido ininterruptamente na televisão da sala de espera do hospital.
O projeto, visando o tripé ensino-pesquisa-extensão, conta com o desenvolvimento de pesquisas, artigos científicos e apresentações de trabalhos em congressos nacionais e internacionais. Nesse contexto, são realizadas reuniões quinzenais da equipe, nas quais se discute a evolução do projeto, o papel de cada um no desenvolvimento das ações e outros tópicos de interesse. Além disso, a equipe também participa de palestras ministradas por profissionais de outras áreas da saúde como, por exemplo, da enfermagem obstétrica, os quais apresentam conteúdo relevante ao aprimoramento do projeto e padronização da atenção, principalmente com relação ao aleitamento materno exclusivo.

\section{A Experiência}

O projeto foi aprovado pelo Comitê de Ética em Pesquisa com Seres Humanos (parecer $n^{\circ} 1.055 .927 / 2015$ ) e conta com três frentes de atuação. A primeira tange a abordagem educativa e preventiva das gestantes, realizada exclusivamente pelos acadêmicos de graduação no hospital universitário, enquanto as gestantes aguardam avaliação médica. Nessa frente, os acadêmicos orientam as gestantes a fim de desmistificar crenças sobre gravidez e tratamento odontológico, bem como conscientizá-las de seu papel na aquisição e manutenção da saúde bucal e geral da criança. As abordagens são realizadas inicialmente em grupos, seguidas por abordagem individual, onde é realizada a anamnese e orientações de higiene, assim como esclarecimento de dúvidas. As gestantes que necessitam de manejo clínico são atendidas no ambulatório de saúde bucal do hospital, ou encaminhadas para as clinicas odontológicas da universidade.

Os tópicos da primeira frente são abordados de acordo com o trimestre da gestante e reforçados a cada consulta pré-natal. Nesse primeiro momento, os principais tópicos envolvem: relação de hábitos e estilo de vida com a aquisição de saúde; conscientização sobre problemas bucais e seus riscos; informações sobre os efeitos de drogas, cigarro e álcool; automedicação; importância do controle de biofilme, e seu risco no desenvolvimento de problemas periodontais, e sua conexão com parto prematuro e o baixo peso do bebê (IHEOZOR-EJIOFOR et al., 2017, MEGA et al., 2017); importância de hábitos alimentares saudáveis; importância do aleitamento materno nos aspectos nutricionais, psicológicos e de desenvolvimento estomatognático do bebê; amamentação exclusiva até os seis meses de idade; o teste de linguinha e sua relevância no desenvolvimento do bebê; sucção não-nutritiva; orientação para a realização da higiene bucal no bebê edêntulo; sintomatologia e cronologia do processo de erupção; como fazer a higiene bucal antes e após a erupção; orientação sobre o uso adequado do flúor e o momento da primeira consulta ao odontopediatra.

No segundo momento, os acadêmicos atuam em conjunto com as residentes em odontologia hospitalar neonatal do hospital universitário. Nessa frente, a abordagem é realizada após o parto, no puerpério imediato. As 
puérperas recebem reforço nas orientações sobre o aleitamento materno e hábitos de sucção não-nutritiva. Neste cenário, enquanto elemento de um processo assistencial preventivo no momento puerperal, são realizadas orientações sobre o manejo da pega correta para o aleitamento materno, visando diminuir o número de puérperas que suspendem o aleitamento materno por motivos como falta de conhecimento, "leite fraco", ferimentos e dor nas mamas e recusa do bebê em pegar o peito (ARAÚJO et al., 2008). Ainda neste momento, é realizado o teste de linguinha, seguindo protocolo sugerido por Martinelli et al. (2013) e o Bristol Tongue Assessment Tool - BTAT (INGRAM et al., 2015), o qual abrange história clínica, avaliação anatomofuncional e sucção nutritiva. Os recém-nascidos (RN) diagnosticados com anquiloglossia são submetidos à liberação cirúrgica do frênulo lingual. Caso o RN apresente dificuldade na amamentação, esse é agendado para retorno, entre 10 e 15 dias, ao ambulatório de saúde bucal. Nesse momento, as mães são convidadas a participarem do projeto de acompanhamento da saúde bucal na primeira infância, com respectivo agendamento.

A terceira frente de atuação é realizada no ambulatório de saúde bucal materno-infantil pelos acadêmicos e residentes. São atendidos os RN de retorno, sendo esses acompanhados trimestralmente até um ano de idade, conforme o protocolo desenvolvido pelo projeto. Aos três meses, as mães recebem orientações sobre irrupção dentária, higiene bucal, hábitos de sucção não-nutritiva e introdução de alimentos a partir dos seis meses. Aos seis meses, é realizada novamente orientação sobre a alimentação adequada, higiene bucal, uso de flúor, hábitos de sucção não-nutritiva, doença cárie e trauma, as quais são reforçadas no terceiro e último encontro.

\section{Discussão}

A importância do pré-natal odontológico e manutenção de saúde durante a gravidez tem sido abordada pela literatura nacional e internacional (BASTIANI et al., 2010, GEORGE et al., 2012). Os estudos têm demonstrado que a baixa procura por esse serviço está principalmente relacionada à falta de conhecimento sobre as implicações da saúde bucal ineficiente sobre a gravidez, assim como limitações no acesso, falta de conhecimento público da relação odontologia-gravidez, mitos persistentes e preocupações com a exposição e segurança do feto ao procedimento propriamente dito (GEORGE et al., 2012). Uma das manifestações periodontais comuns durante a gestação é o aumento da inflamação gengival sem, contudo, haver aumento dos níveis de biofilme (KRÜGER et al., 2017). É importante salientar que, mesmo que as evidências cientificas não indiquem claramente a relação entre doença periodontal (DP) e parto prematuro/baixo peso ao nascimento (POLYZOS et al., 2010), a educação e prevenção em saúde bucal podem garantir uma redução no número de microrganismos bucais, consequentes mediadores de inflamação, além de permitir aquisição de novos conhecimentos sobre sua saúde bucal e cuidados para com o seu bebê, melhorando hábitos e comportamentos, que consequentemente irão garantir benefícios à gestante e ao seu filho (REIS et al., 2010).

O projeto contribui com as ações de políticas públicas de saúde voltadas para a atenção integral das mulheres durante a fase de gravidez, parto, puerpério, e para a criança nos primeiros anos de vida (EUZÉBIO et al., 2013), assim como contempla a experiência acadêmica em nível hospitalar, a qual é constantemente relatada pelos participantes como excelente para o aprofundamento do conhecimento, suprindo assim a lacuna que existe nos currículos odontológicos, no que tange a presença de disciplinas voltadas a odontologia hospitalar. Diante da complexidade do ser humano, a integração do acadêmico com o individuo hospitalizado cria uma janela para a formação de profissionais preocupados em atender o paciente de uma forma mais segura e ter uma visão mais ampla, buscando aprimorar seu conhecimento para a atuação neste cenário (EUSÉBIO et al., 2013). A resolução $n^{\circ} 3$ do CNE/CSE, de 2002, inseriu novas diretrizes curriculares para os cursos de graduação, as quais visam uma formação mais humanista, crítica e reflexiva, para a atuação em todos os níveis de atenção em saúde (OLIVEIRA JUNIOR et al., 2017).

De fato, a educação da população em saúde tem sido intensificada por projetos de extensão. Oliveira Junior et al. (2017) identificaram, em uma atividade extensionista, que usuários de um Restaurante Popular da cidade de Patos (Paraíba - BR) são carentes no que se refere a informações sobre saúde bucal. Os autores relatam que o projeto motivou o autocuidado por parte dos frequentadores do recinto, assim como gerou um ambiente de educação para saúde. Ao mesmo tempo, demarcam a importância que o projeto teve na formação do acadêmico como profissional promissor. O relato corrobora as experiências do presente projeto de extensão, no qual as gestantes têm a oportunidade de adquirir conhecimento sobre a importância da saúde bucal.

Dentre as limitações durante a execução do projeto, podemos citar a resistência de algumas gestantes e de outros profissionais da saúde no desenvolvimento do projeto, uma vez que a relação entre odontologia e o período gravídico-puerperal ainda não é clara para gestantes e para estes profissionais. Espera-se, com as novas diretrizes curriculares das diferentes áreas da saúde, uma abordagem integradora, difundindo assim mais conhecimento. Outro aspecto a ser destacado envolve a alta necessidade de procedimentos curativos que tem sido encontrada neste público, o que remete a importância da abordagem educacional em saúde bucal, focada principalmente na prevenção e elaboração de um plano de ação para capacitação dos profissionais envolvidos ao atendimento odontológico das gestantes.

Enfim, por meio do presente projeto foi possível constatar que a extensão universitária em âmbito hospitalar, com um público específico, possibilitou a criação de um vínculo entre as gestantes/puérperas e os acadêmicos/residentes, além de uma de experiência 
interdisciplinar, que resultou em uma melhora na conscientização da mãe sobre o seu papel na saúde do bebê e da família, alavancando também um desenvolvimento intelectual dos envolvidos, possibilitando a procura pela assistência odontológica durante a gravidez e logo após o nascimento. Os acadêmicos, por sua vez, relataram que a experiência é extremamente importante para a consolidação dos conhecimentos necessários para sua formação integradora.

\section{Conclusões}

O acompanhamento durante o período gravídicopuerperal por um cirurgião-dentista é necessário tanto para garantir a aquisição e manutenção da saúde bucal da mãe, assim como um desenvolvimento apropriado do bebê. Acredita-se que as limitações para a incorporação prática cotidiana do manejo de gestantes e puérperas e primeira infância dependem de fatores emocionais, ambientais e culturais, tanto dos cirurgiões-dentistas quanto dos pacientes, fatores que tornam a prática do aleitamento materno, consulta pré-natal odontológica, teste da linguinha e manutenção da saúde bucal, grandes desafios para esse público. Conclui-se que o empenho dos acadêmicos, residentes e mestrandos nessa atividade interdisciplinar de educação em saúde, conduz a benefícios educacionais para alunos e população, em especial as gestantes, incentivando a continuidade e o aprimoramento do projeto.

\section{Contribuição de cada autor}

Os autores A.T.G., N.P., A.R.S., B.R.S. participaram da redação inicial; M.C.P., J.T.D., N.S.G., F.B.T.A. planejaram o projeto; M.C.P. e F.B.T.A. realizaram a revisão intelectual crítica do artigo e a redação final; e F.B.T.A atuou como coordenadora e orientadora dos bolsistas.

\section{Referências}

ARAÚJO, O. D.; CUNHA, A. L. D.; LUSTOSA, L. R.; NERY, I. S.; MENDONÇA, R. C. M.; CAMPELO, S. M. A. Aleitamento materno: fatores que levam ao desmame precoce. Revista Brasileira de Enfermagem, v. 61, n. 4, p. 488-492, 2008.

BASTIANI, C.; COTA, A. L. S.; PROVENZANO, M. G. A.; FRACASSO, M. D. L. C.; HONÓRIO, H. M.; RIOS, D. Conhecimento das gestantes sobre alterações bucais e tratamento odontológico durante a gravidez. Odontologia Clínico-Científica, v. 9, n. 2, 2010.

BUSS, P. M. Promoção da saúde e qualidade de vida. Ciência \& Saúde Coletiva, v. 5, n. 1, p. 163-177, 2000.
EUSÉBIO, L. F.; VIANA, K. A.; CORTINES, A. A. de O.; COSTA, L. R. Atuação do cirurgião-dentista em equipe multiprofissional de atenção hospitalar à Saúde Materno-Infantil. Revista Odontológica do Brasil Central, v. 21, n. 60, p. 16-20, 2013.

GEORGE, A; SHAMIM, S.; JOHNSON, M.; DAHLEN, H.; AJWANI, S.; BHOLE, S.; YEO, A. E. How do dental and prenatal care practioners perceive dental care during pregnancy? Current Evidence and Implications. Birth, v. 39, n. 3, p. 238-247, 2012.

IHEOZOR-EJIOFOR, Z; MIDDLETON, P.; ESPOSITO, M.; GLENNY, A. M. Treating periodontal disease for preventing adverse birth outcomes in pregnant women. The Coachrane Database of Systematic Reviews, v. 12, n. 6, art. CD005297, 2017.

INGRAM, J.; JOHNSON, D.; COPELAND, M.; CHURCHILL, C.; TAYLOR, H.; EMOND, A. The development of a tongue assessment tool to assist with tongue-tie identification. Archives of Disease in Childhood Fetal and Neonatal, v. 100, n. 4, p. 344-349, 2015.

KRÜGER, M. S. D. N.; CASARIN, R. P.; GONÇALVES, L. B.; PAPPEN, F. G.; BELLOCORREA, F. O.; ROMANO, A. R. Periodontal Health Status and Associated Factors: Findings of a Prenatal Oral Health Program in South Brazil. International Journal of Dentistry, v. 2, p. 1-6, 2017.

MARTINELLI, R. L. C.; MARCHESAN, I. Q.; BERRETIN-FELIX, G. Protocolo de avaliação do frênulo lingual para bebês: relação entre aspectos anatômicos e funcionais. Revista CEFAC, v. 15, n. 3, p. 599-610, 2013.

MEGA, K.; DRAGIDELlA, F.; DISHA, M.; SLLAMNIKU-DALIPI, Z. The Association between Periodontal Disease and Preterm Low Birthweight in Kosovo. Acta Stomatologica Croatica, v. 51, n. 1, p. 33-40, 2017.

MOIMAZ, S. A. S.; ROCHA, N. B.; SALIBA, O.; GARBIN, C. A. S. O acesso de gestantes ao tratamento odontológico. Revista de Odontologia da Universidade Cidade de São Paulo, v. 19, n. 1, p. 39-45, 2007.

OLIVEIRA JÚNIOR, J. K. et al. Práticas educativas em saúde bucal direcionadas aos usuários do restaurante popular da cidade de Patos-PB. Revista Brasileira de Extensão Universitária, v. 8, n. 2, p. 67-74, 2017.

POLYZOS, N. P. et al. Obstetric outcomes after treatment of periodontal disease during pregnancy: systematic review and meta-analysis. The BMJ, v. 341, art. c7017, 2010.

REIS, D. M.; PITTA, D. R.; FERREIRA, H. M. B.; DE JESUS, M. C. P.; DE MORAES, M. E. L.; SOARES, M. G. Educação em saúde como estratégia de promoção de saúde bucal em gestantes. Ciência \& Saúde Coletiva, v. 15, n. 1, p. 269-276, 2010.

SANTOS NETO, E. T.; OLIVEIRA, A. E.; ZANDONADE, E.; LEAL, M. C. Acesso à assistência 
odontológica no acompanhamento pré-natal. Ciência \& Saúde Coletiva, v. 17, n. 11, p. 3057-3068, 2012.

SILVEIRA, J. L. G. C.; GARCIA, V. L. Mudança curricular em Odontologia: significados a partir dos sujeitos da aprendizagem. Interface - Comunicação, Saúde, Educação, v. 19, n. 52, p. 145-158, 2015.

Como citar este artigo:

POMINI, M. C.; GAWLIK, A. T.; PEREIRA, N.; DOS SANTOS, A. R.; DOS SANTOS, B. R.; DEMOGALSKI, J. T.; DE GOUVÊA, N. S.; ALVES, F. B. T. Educação em Saúde Bucal a Gestantes, Puérperas e Primeira Infância: Relato de Atividade de Extensão. Revista Brasileira de Extensão Universitária, v. 8, n. 3, p. 143-148, 2017. Disponível em: < https://periodicos.uffs.edu.br/index.php/RBEU/article/view/586 $\underline{1 / p d f}>$ 